J. Dairy Sci. 99:7049-7052

http://dx.doi.org/10.3168/jds.2016-11280

(C) American Dairy Science Association ${ }^{\circledR}, 2016$.

\title{
Short communication: Presence of Lactococcus and lactococcal exopolysaccharide operons on the leaves of Pinguicula vulgaris supports the traditional source of bacteria present in Scandinavian ropy fermented milk
}

\author{
Davide Porcellato, ${ }^{1}$ Malena Tranvåg, and Judith Narvhus \\ Department of Chemistry, Biotechnology and Food Science, Norwegian University of Life Sciences, PO Box 5003, N-1432 Ås, Norway
}

\begin{abstract}
Some traditional Scandinavian fermented milk products have a pronounced ropy consistency due to the presence of exopolysaccharide-producing strains of Lactococcus lactis ssp. cremoris. Norwegian food folklore describes how leaves from the carnivorous plant Pinguicula vulgaris (common butterwort) may be added to milk to initiate the fermentation of the traditional fermented milk product tettemelk. However, scientific confirmation of the link between the plant and the milk product has not been previously published. In the present study, the microbiome on 20 samples of P. vulgaris leaves collected from 5 different rural geographical locations in Norway and from 4 samples of commercial tettemelk was analyzed using high-throughput sequencing methods. The leaf microbiota of $P$. vulgaris was dominated by Proteobacteria and Firmicutes and the genus Lactococcus was demonstrated in all leaf samples. In addition, DNA extracted from the leaf microbiome contained genes identical to those responsible for exopolysaccharide production in Lactococcus. These results confirm the traditional use of $P$. vulgaris as a source of bacteria for the Norwegian ropy fermented milk product tettemelk and indicate that P. vulgaris microbiomes can be a potential source of lactic acid bacteria with interesting dairy technological features.
\end{abstract}

Key words: Lactococcus, exopolysaccharide, tettemelk, Pinguicula vulgaris

\section{Short Communication}

A type of traditional fermented milk, generically known as "ropy milks," is consumed in northern Scandinavian countries. In Norway, food folklore describes the use of the leaves of Pinguicula vulgaris (common but-

Received April 6, 2016.

Accepted June 5, 2016.

${ }^{1}$ Corresponding author: davide.porcellato@nmbu.no terwort, known as tettegras in Norway) to initiate the fermentation of a ropy (or slimy) fermented milk, known as tettemelk. The methodology for this is not described in detail but apparently involves several subcultures of the original leaf and milk mixture before a satisfactory tettemelk is obtained. However, many attempts to produce this traditional product from the leaves have failed, thus leading to doubt that this is actually a correct interpretation of earlier practices (Narvhus, 2014). Tettemelk is characterized by an extremely viscous consistency, a mild aromatic flavor, and resistance to syneresis. Exopolysaccharide (EPS)-producing strains of Lactococcus lactis ssp. cremoris have been isolated from tettemelk and similar Scandinavian fermented milk products, but unequivocal proof that the bacterium producing the ropy consistency is present on the leaves of P. vulgaris has not been obtained (Narvhus, 2014). Using an artisan starter culture collected from neighboring farms in the Røros district, tettemelk has been commercially produced by Rørosmeieriet since 1996. The culture is propagated and stored at the dairy, and the culture itself is not commercially available. Production of EPS is a key property for the production of several types of fermented milk, and EPS-producing lactic acid bacteria are widely used by dairy companies to improve the consistency of fermented milk products.

The aim of this study was to investigate the possible link between $P$. vulgaris and tettemelk. We analyzed the microbiome on the P. vulgaris leaves and tettemelk using massive parallel sequencing and targeting specific lactococcal genes involved in the production of exopolysaccharides.

Twenty samples of $P$. vulgaris leaves were collected from 5 rural geographical locations in Norway (Lykkja, Hardanger, Damtjern, Vega, and Røros) and 4 cartons of commercial ropy fermented milk (tettemelk) were collected. Leaf samples were homogenized for $2 \mathrm{~min}$ in Ringer's solution (Oxoid, Basingstoke, UK) using an Omni mixer (Omni International, Waterbury, CT). The DNA was extracted from leaf homogenates and commer- 
A

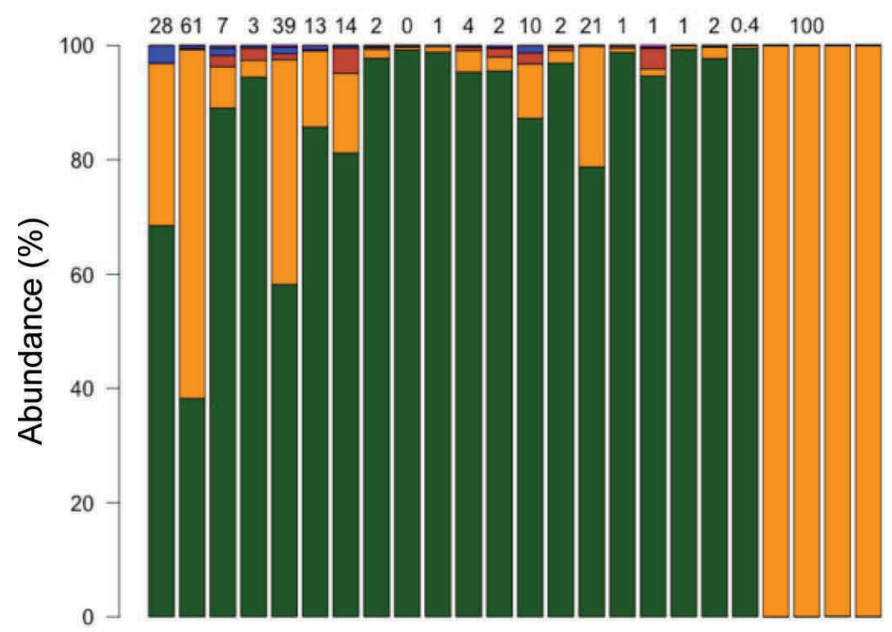

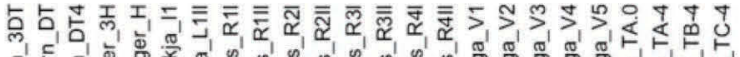

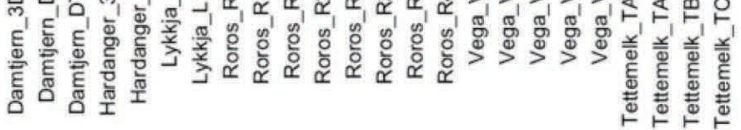

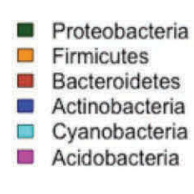

\section{ฮㅇำ}

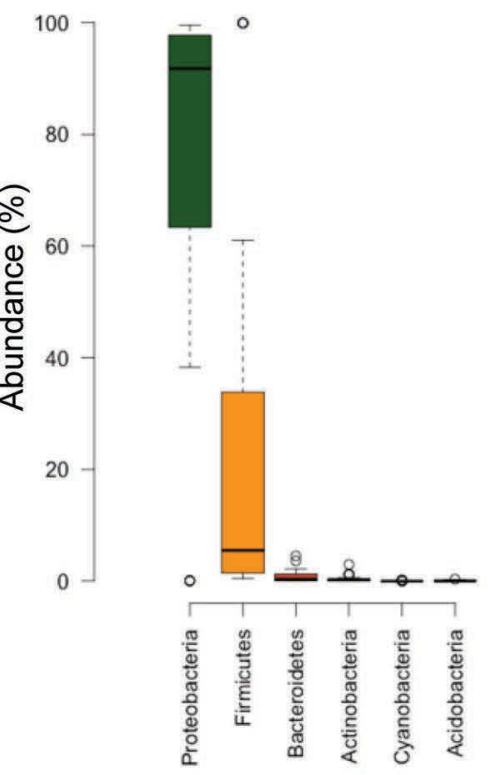

$\mathrm{D}$

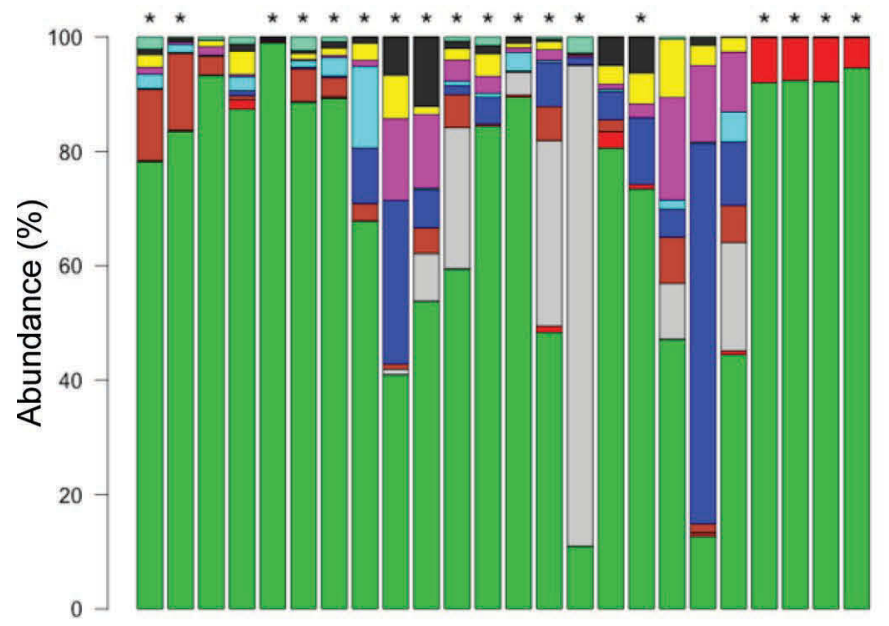

Lactococcus
Leuconostoc
Carnobacterium
Streptococcus
Bacillus
Lactobacillus
Staphylococcus
Uncultured
- Anoxybacillus
Others OTUs
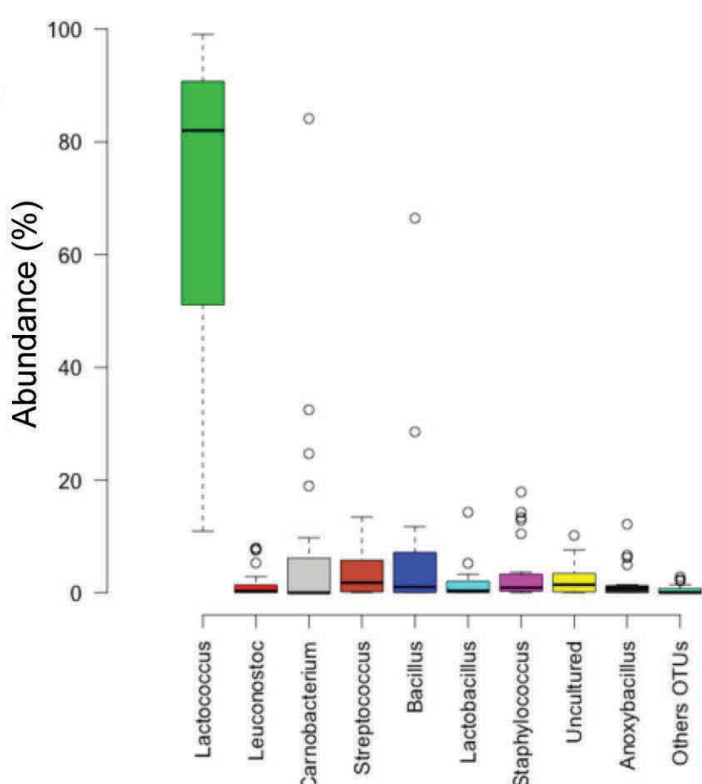

Figure 1. Relative abundance of the bacterial 16S rRNA sequences obtained from Pinguicula vulgaris leaves and traditional fermented milk. (A) Percentage abundance of bacterial phyla in all the samples. Numbers over the barplot indicate the percentage of Firmicutes in each sample. The 4 samples of fermented milk (tettemelk) contained 100\% Firmicutes. (B) Boxplot of the 6 phyla identified in leaves and fermented milk microbiomes. (C) Percentage abundance of genus within the Firmicutes phyla for all the samples of leaves and fermented milk. $*$ indicates the presence of lactococcal exopolysaccharide operons in the sample. (D) Boxplot of the 10 most abundant genera within the Firmicutes phylum. $\mathrm{OTU}=$ operational taxonomic unit. Color version available online. 
cial samples as previously described (Porcellato et al., 2014), and amplification of the V3 and the V4 regions of the bacterial $16 \mathrm{~S}$ rRNA gene was performed using the universal primer Uni340F (5'-CCTACGGGRBGCASCAG-3') and Bac806R (5'-GGACTACYVGGGTATCTAAT-3'; Takai and Horikoshi 2000). Unique 6-bp barcodes were used to identify each library. Massive parallel sequencing was performed on an Illumina Miseq (Illumina, San Diego, CA). Raw files were demultiplexed, and forward and reverse reads were merged using Qiime 1.9.0 (Caporaso et al., 2010). Reads were quality filtered using Usearch 8.0 (Edgar, 2010) and operational taxonomic units (OTU) were picked using the UPARSE OTU algorithm (Edgar, 2013) clustered at $97 \%$ pairwise identity. Taxonomy was assigned to each OTU representative sequence after aligning each sequence against the Silva database (Quast et al., 2013) using the blastn algorithm (Camacho et al., 2009). The 20 best hits for each OTU representative sequence were extracted, and the hits with lowest e-value and highest identity were used to assign the taxonomy.

The microbiota composition obtained from the 20 leaf samples and from the fermented milk consisted of 166 OTU clustered at $97 \%$ similarity. Permutational multivariate ANOVA using Unifrac distance matrix was used to compare microbial communities of groups of samples. The fermented milk samples showed a simple microbiota consisting of approximately $90 \%$ Lactococcus and 10\% Leuconostoc (Figure 1), whereas the average OTU richness of the leaves was $50 \pm 21$ OTU. The phyla present on the leaves in greatest abundance were Proteobacteria (88\% of all the reads) and Firmicutes (10\%; Figures 1A and 1B). Among the Firmicutes, the genus Lactococcus was the most abundant OTU detected ( $75 \%$ of the reads assigned to Firmicutes) and was detected in all the samples, with abundance varying from 0.2 to $51 \%$ of the total reads (Figure 1). Other abundant OTU of the phylum Firmicutes on the leaf samples were Carnobacterium (8.6\%), Streptococcus $(7.4 \%$ and present in all the samples), Bacillus (2.6\%), and Staphylococcus $(1.4 \%)$ as shown in Figures $1 \mathrm{C}$ and 1D. The microbiota of the fermented milk comprised 2 genera of the phylum Firmicutes found on the leaves, namely Lactococcus and Leuconostoc (Figures 1A and 1C). Leuconostoc was detected in 10 leaf samples and in low abundance (from 0.01 to $0.16 \%$ ). Microbiota composition was also compared using nonmetric multidimensional scaling. Clear separation on the NMDS plot was detected for the leaf samples and the fermented milk (Figure 2). However, this result was not unexpected since the development

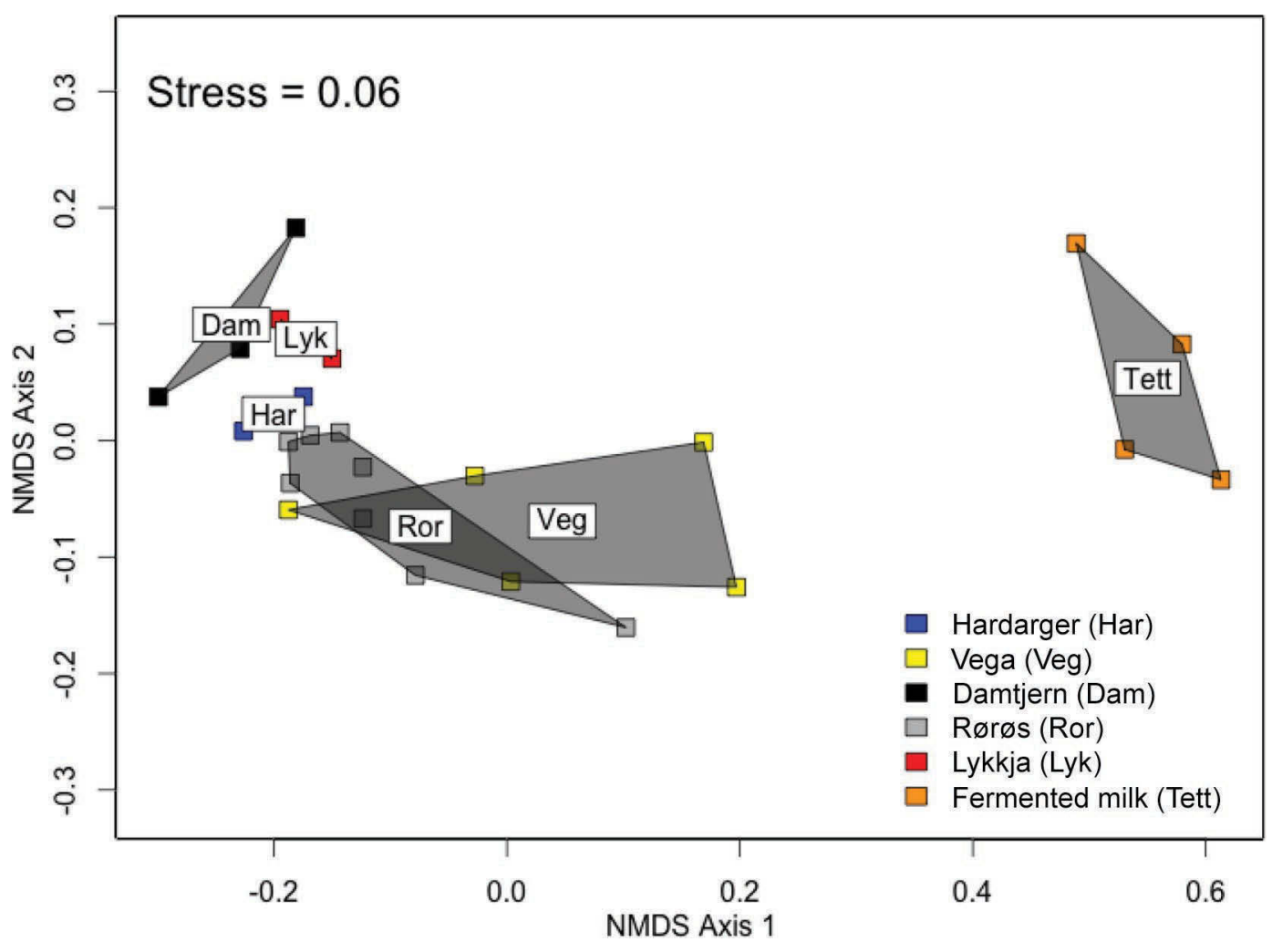

Figure 2. Nonmetric multidimensional scaling (NMDS) plot using unweighted Unifrac-distance matrix for all the $16 \mathrm{~S}$ amplicon libraries of leaf and fermented milk samples. Samples are grouped by place and category. Color version available online. 
of a good tettemelk culture from the leaves is reputed to require several subcultures in milk. Considering the mixed microbiota on the leaves, this subculturing could lead to the gradual domination of acid-forming lactic acid bacteria over the acid-sensitive Proteobacteria.

To investigate whether Lactococcus EPS genes were present on the leaf samples and fermented milk, new primers targeting the lactococcal EPS operon were designed. Ten Lactococcus EPS operons (downloaded from National Center for Biotechnology Information, GenBank accession number CP009472, CP003132, AF142639.1, EF192212, AF036485, AE005176, CP006766, CP009054, AY741550, and HQ665557) and 3 operons obtained from genome sequences of laboratory strains of Lactococcus lactis ssp. cremoris were used to design EPS operon primers, which amplify a large fragment $(>1,000 \mathrm{bp})$. The primers used in this study were EPS380F (5'-AGAAAGGGAATTGGGTTACCCT-3') and EPS1623R (5'-TGAGGCTGTGTAAACTGGGG-3'). The PCR products were separated by gel electrophoresis and resulting bands were sequenced and identified as equivalent to the Lactococcus lactis spp. cremoris EPS operon. Fourteen of the 20 leaf samples were positive for EPS operons as well as all the 4 samples of fermented milk (indicated by a star above the columns representing each sample in Figure 1C).

Although lactococci have been isolated from a variety of plant environments, Lactococcus lactis ssp. cremoris is rarely found outside the dairy environment. It has been suggested that industrial dairy strains are descended from lactococci found on plants and have undergone adaptation to the dairy environment (Cavanagh et al., 2015). In this study, lactococci were demonstrated to be part of the microbiome in all 20 leaf homogenates and 14 of these homogenates showed the presence of Lactococcus and the lactococcal EPS operon. Thus, we have shown that the leaves of Pinguicula vulgaris are a source of EPS-producing lactococci, thereby confirming the traditional use of these leaves to make tettemelk, as described in Norwegian food folklore.

\section{ACKNOWLEDGMENTS}

The authors express thanks to TINE SA (Oslo, Norway) for funding and to Rørosmeieriet AS (Røros, Norway) for providing the fermented milk products.

\section{REFERENCES}

Camacho, C., G. Coulouris, V. Avagyan, N. Ma, J. Papadopoulos, K. Bealer, and T. L. Madden.2009. BLAST plus: Architecture and applications. BMC Bioinformatics 10:421.

Caporaso, J. G., J. Kuczynski, J. Stombaugh, K. Bittinger, F. D. Bushman, E. K. Costello, N. Fierer, A. G. Peña, J. K. Goodrich, J. I. Gordon, G. A. Huttley, S. T. Kelley, D. Knights, J. E. Koenig, R. E. Ley, C. A. Lozupone, D. McDonald, B. D. Muegge, M. Pirrung, J. Reeder, J. R. Sevinsky, P. J. Turnbaugh, W. A. Walters, J. Widmann, T. Yatsunenko, J. Zaneveld, and R. Knight. 2010. QIIME allows analysis of high-throughput community sequencing data. Nat. Methods 7:335-336.

Cavanagh, D., G. F. Fitzgerald, and O. McAuliffe. 2015. From field to fermentation: The origins of Lactococcus lactis and its domestication to the dairy environment. Food Microbiol. 47:45-61.

Edgar, R. C. 2010. Search and clustering orders of magnitude faster than BLAST. Bioinformatics 26:2460-2461.

Edgar, R. C. 2013. UPARSE: Highly accurate OTU sequences from microbial amplicon reads. Nat. Methods 10:996-998.

Narvhus, J. 2014. Northern European fermented milk. Pages 895-899 in Encyclopedia of Food Microbiology. C. Batt and C. A. Batt, ed. Elsevier Academic Press, New York, NY.

Porcellato, D., C. Brighton, D. J. McMahon, C. J. Oberg, M. Lefevre, J. R. Broadbent, and J. L. Steele. 2014. Application of ARISA to assess the influence of salt content and cation type on microbiological diversity of Cheddar cheese. Lett. Appl. Microbiol. 59:207-216.

Quast, C., E. Pruesse, P. Yilmaz, J. Gerken, T. Schweer, P. Yarza, J. Peplies, and F. O. Glöckner. 2013. The SILVA ribosomal RNA gene database project: Improved data processing and web-based tools. Nucleic Acids Res. 41:D590-D596.

Takai, K., and K. Horikoshi. 2000. Rapid detection and quantification of members of the archaeal community by quantitative PCR using fluorogenic probes. Appl. Environ. Microbiol. 66:5066. 